\title{
Preliminary simulations of internal waves and mixing generated by finite amplitude tidal flow over isolated topography
}

\author{
Sonya Legg ${ }^{1,2}$ and Karin M.H. Huijts ${ }^{3}$ \\ ${ }^{1}$ Department of Physical Oceanography, \\ Woods Hole Oceanographic Institution, Woods Hole, MA 02543 \\ ${ }^{2}$ Program in Atmosphere and Ocean Sciences \\ Princeton University, Princeton, NJ 08544 \\ ${ }^{3}$ Institute for Marine and Atmospheric Research \\ Utrecht University, \\ Utrecht, The Netherlands
}

Submitted to Deep Sea Research

September 29, 2005 


\section{Abstract}

Much recent observational evidence suggests that energy from the barotropic tides may be used for mixing in the deep ocean. Here the process of internal tide generation and dissipation by tidal flow over an isolated Gaussian topography is examined, using 2-dimensional numerical simulations employing the MITgcm. Four different topographies are considered, for five different amplitudes of barotropic forcing, thereby allowing a variety of combinations of key nondimensional parameters. While much recent attention has focused on the role of relative topographic steepness and height in modifying the rate of conversion of energy from barotropic to baroclinic modes, here attention is focused on parameters dependent on the flow amplitude. For narrow topography, large amplitude forcing gives rise to baroclinic responses at higher harmonics of the forcing frequency. Tall narrow topographies are found to be the most conducive to mixing. Dissipation rates in these calculations are most efficient for the narrowest topography.

Keywords: Tides, internal waves, ocean mixing.

\section{Introduction}

The tides are now accepted as one of the most significant sources of energy for mixing in the ocean interior (along with the winds) (Munk and Wunsch, 1998). While the dominant mechanism of tidal mixing on many continental shelves, the turbulent frictional boundary layer, is relatively well understood, in the deep ocean a complex series of steps are required to move energy from the barotropic flow into the small scales where mixing can occur. These steps can be summarized as: (1) conversion of barotropic energy into baroclinic energy as stratified fluid is pushed over topographic obstacles; (2) mixing local to the topography by that part of the baroclinic flow with sufficiently high shear; (3) radiation of energy away from the topography by that part of the baroclinic flow in the form of internal waves; (4) non-linear wave-wave interactions causing the cascade of energy to smaller scales; (5) wave-topography interactions leading to further cascade of energy to small 
scales; (6) mixing when shear is sufficiently high (i.e. energy is at sufficiently small vertical lengthscales). Each of these steps has received significant attention in the past few years. In particular good progress has been made in understanding the dependence of the conversion of barotropic to baroclinic energy on topographic steepness (Balmforth et al., 2002; Llewellyn Smith and Young, 2002; St Laurent et al., 2003), and in detailing the "wave turbulence" that leads to the cascade to smaller scales (Y.V. Lvov, 2004; Polzin, 2004). Munroe and Lamb (2005) and Holloway and Merrifield (1999) have shown that conversion rates are modified when topography is 3-dimensional rather than a 2-dimensional ridge. The study of wave breaking through reflection from topography is ongoing (Legg and Adcroft (2003); Zikanov and Slinn (2001); Nash et al. (2004)). Little however is known about the processes which determine the partitioning of the baroclinic tidally-generated flow between waves and motions leading to local mixing at the topography. This preliminary study is designed to make a first attempt to answer this question, focusing on a simple idealized 2-dimensional topographic shape in a quiescent ocean, and examining the waves and dissipation produced for a selection of topographic parameters as the barotropic tidal speed is progressively increased.

An ultimate goal of research into tidal mixing is the development of a physically based, energetically consistent parameterization of diapycnal mixing in the ocean interior, a process of key importance in the global thermohaline circulation. Recently a first attempt was made to formulate such a parameterization (Simmons et al., 2003). In the absence of sufficient knowledge about the energy partition between mixing and waves, the authors simply assumed 1/3 of energy was dissipated locally to the topography, and the other $2 / 3$ were radiated away as waves. This study is designed to begin to refine this estimate.

The key physical parameters governing the response to tidal flow over topography are: (a) $U_{0}$, the amplitude of the barotropic tide; (b) $\omega_{0}$, the frequency of the barotropic tide; (c) $f$, the coriolis frequency; (d) $N$, the buoyancy frequency, (e) $h_{0}$, the topographic height, (f) $L$, the topographic length scale, (g) $H$, the total water depth. From these parameters, we have a total of 5 independent nondimensional parameters. One possible choice of nondimensional parameters is: (a) $U_{0} /\left(\omega_{0} L\right)=R_{L}$, the tidal excursion parameter; (b) $h_{0} / L$ or $d h / d x$, the topographic slope; (c) $\left[\left(\omega_{0}^{2}-f^{2}\right) /\left(N^{2}-\omega_{0}^{2}\right)\right]^{1 / 2}=s$, the internal wave characteristic slope; (d) $h_{0} / H=\delta$, the relative height of the topography; (e) $U_{0} /\left(N h_{0}\right)=F r$, the Froude number of the flow. In this study we will 
vary $U_{0}, h_{0}$ and $L$, thereby varying all nondimensional parameters except $s$.

Of these nondimensional parameters, most attention recently has focused on the combination $\gamma=(d h / d x) / s$, the relative steepness of the topography when compared to the internal wave slope. When $\gamma<1$, slopes are subcritical, while when $\gamma>1$, the slope is supercritical. Earlier studies (Bell, 1975) focused on the conversion of barotropic to baroclinic energy by flow over subcritical topography; recent studies have extended understanding into the supercritical regime, analytically for both $\gamma \rightarrow 1$ (Balmforth et al., 2002) and for $\gamma=\infty$ (St Laurent et al., 2003), and numerically for $\gamma>1$ (Khatiwala, 2003). For steep slopes and deep fluid, energy conversion is enhanced by a factor 2, relative to predictions made assuming subcritical slopes (St Laurent et al., 2003; Llewellyn Smith and Young, 2003).

The studies of Bell (1975) assumed $\gamma<<1$, but examined the role of finite amplitude $R_{L}=$ $U_{0} /\left(\omega_{0} L\right)$, the tidal excursion parameter. For small $R_{L}$, the response is entirely at the forcing frequency $\omega_{0}$, but for $R_{L}>1$, waves at higher harmonic frequencies $n \omega_{0}$ are generated. For large $R_{L}$ the quasi-steady limit applies, in which the lee-waves have the intrinsic frequency $U_{0} / L$. The recent studies mentioned above, while they examined increasing $\gamma$, all assumed the response was at the forcing frequency, hence presuming $R_{L}<1$ (even for the knife-edge slope in St Laurent et al. (2003), which must by definition have infinitely small $L$ and hence large $R_{L}$ ).

The role of finite ocean depth, as measured by $\delta=h_{0} / H$ has been considered by several authors (Llewellyn Smith and Young, 2002; Khatiwala, 2003; St Laurent et al., 2003). They find that for small $\gamma$, increasing $\delta$ leads to a decrease in the energy conversion rate compared to the infinite depth limit. St Laurent et al. (2003) finds that as $\delta \rightarrow 1$, the enhancement of the conversion rate induced by steep topography increases greatly compared to the factor of 2 seen for steep topography in an infinitely deep fluid.

Finally the Froude number parameter $U_{0} /\left(h_{0} N\right)$ is a measure of the impediment of the topography to the flow. For large Fr, the flow is relatively unaffected by the topography, whereas for small Fr the flow is blocked by the topography. Nycander (2005) outlines the regimes delimited by Fr: for large $R_{L}$ (i.e. the quasi-steady flow limit (Bell, 1975)) the flow response to the topography is linear if $\mathrm{Fr}>>1$. The most interesting regime is at intermediate Fr, when locally the flow may transition from a subcritical to a supercritical state as it passes over the topography. Simulations in Legg (2004) showed evidence for transient hydraulic effects at $F r \approx 1$. 
We expect mixing to occur when shears are large, which we would expect to be more likely if the velocity amplitudes of the internal tides are large. Hence mixing might be more likely for higher $U_{0}$. Similarly, local mixing might be more likely if there are local internal hydraulic effects at the topography, e.g. a sub- to super-critical flow transition and downstream hydraulic jump. For this reason we are motivated to examine the hitherto neglected area of the response of baroclinic flow to finite amplitude barotropic flow. This study therefore focuses on the two velocity dependent parameters $U_{0} /\left(\omega_{0} L\right)$ and $U_{0} /\left(h_{0} N\right)$, by varying $U_{0}$. In order to examine different regimes (e.g. high $R_{L}$ combined with low $F r$ and vice versa), different topographic shapes are considered, so that two different values of $h_{0} / H$ are examined, and for each $h_{0} / H$, two different values of $\gamma$ are examined. Our solutions therefore consider variations in 4 of the nondimensional parameters listed above, with only $s$, the wave slope, remaining constant for all calculations. A dominant question is: Are there specific regimes of $R_{L}$ and $F r$ which are more conducive to local mixing, and how do these depend on the topographic height $h_{0} / H$ and steepness $\gamma$ ?

\section{Model configuration and simulation design}

The behavior of flows at large velocity amplitude, with possible overturning and mixing, is intractable analytically, and therefore our tool for this study is numerical simulation, using the nonhydrostatic MITgcm (Marshall et al., 1997). In this preliminary investigation we focus on 2-dimensional simulations, and restrict ourselves to a single Gaussian topography of the form:

$$
h=h_{0} \exp \left(\frac{-\left(x-x_{0}\right)^{2}}{2 L^{2}}\right)
$$

An oscillating barotropic flow in the x-direction of the form

$$
U=U_{0} \sin \left(\omega_{0} t\right)
$$

is imposed uniformly throughout the domain through a body forcing term as described in Khatiwala (2003), with a forcing frequency $\omega_{0}=1.41 \times 10^{-4} s^{-1}$, representing the M2 tide.

Radiative boundary conditions are applied to the baroclinic component of the flow, as described in Khatiwala (2003), to allow internal waves to escape the domain. The fluid is initially stably stratified with a horizontally and vertically uniform stratification with buoyancy frequency 
$N=8 \times 10^{-4} s^{-1}$. The Coriolis frequency is held fixed at $f=8 \times 10^{-5} s^{-1}$. Since $N>\omega_{0}>f$ we are purposely ignoring complications such as critical latitudes, critical levels, and parametric subharmonic instability which ultimately must be included in any global tidal mixing parameterization. Depth variations in stratification would also modify the path of the wave rays, and alter the vertical structure of internal wave modes. For the sake of simplicity we are also ignoring other components of the tidal forcing, since the response to multiple frequency forcing cannot be understood without first understanding the response to a single-frequency forcing.

The flow is initially at rest. The Boussinesq, nonhydrostatic form of the MITgcm is employed, with a linear equation of state. Stratification is dependent on temperature alone. Stress-free velocity boundary conditions and no-flux temperature boundary conditions are applied at the topography and free surface.

In this preliminary study dissipation is effected through a Laplacian friction term in the momentum equations with constant coefficients: $\nu_{h}=1 \mathrm{~m}^{2} / \mathrm{s}, \nu_{v}=0.1 \mathrm{~m}^{2} / \mathrm{s}$. The values of these coefficients were chosen empirically to be sufficient to eliminate grid-scale noise in the velocity fields, and are similar to values used in Legg (2004) for similar resolution. (Note that we have found that problems with rapid temporal variations driven by the tides require larger viscosities than problems dominated by geostrophic and subinertial flows.) For tracers a flux-limiter advection scheme is used (Pietrzak, 1998); this scheme preserves fronts and prevents spurious overshoots in the value of tracers, while also introducing numerical diffusion where needed for stability. We therefore set the explicit tracer diffusivity to zero, which also ensures that the background stable stratification is maintained in the absence of flow, and not eroded by diffusion near the boundaries. A downside to the use of numerical diffusivity is that it is difficult to quantify the diffusive fluxes taking place.

Simulations are carried out for 4 different bottom topographies, corresponding to two different topographic heights, and two different widths at each height. We refer to the topographies as "low,wide", "low,narrow", "tall,wide" and "tall,narrow" respectively. For each topography a total of 5 different calculations are carried out, with $U_{0}$ varying from $2 \mathrm{~cm} / \mathrm{s}$ to $32 \mathrm{~cm} / \mathrm{s}$. Velocities at the lower end of this range are more typical of tides in mid-ocean scenarios, while those at the higher end may be found in coastal regions. In total therefore we have 20 different simulations. Details of the topographic parameters are given in the Table. The "low,wide" topography is always 
subcritical, while the other topographies have maximum slopes which are supercritical.

Our choice of topographic scenarios spans many of the regimes where observations of internal tides have been made. For example, the large-scale structure of the Mid-Atlantic ridge has a subcritical slope, like our "low, wide" topography, while the smaller features found on this slope may be narrow and have supercritical slope like our "low, narrow" topography (St Laurent and Nash, 2004). The Hawaiian ridge is similar in character to our "tall, wide" topography, which has a supercritical slope but relatively large horizontal scale (St Laurent and Nash, 2004; Klymak et al., 2005), while the Knight inlet sill is similar to our "tall, thin" topography, with large amplitude changes in topography in a very short distance (Klymak and Gregg, 2004). Of course a significant difference between our simulations and real ocean locations is the constant stratification and absence of pycnocline in the simulations. The choice of tidal forcing velocities ranges from weak values seen in deep ocean locations to very high values likely only found in coastal locations such as Knight Inlet.

Figure 1 shows the values of $R_{L}$ and $F r$ as a function of $U_{0}$ for each different topography. For the "low,wide" and "tall,wide" topographies, $R_{L}$ is always less than 1 , so that the response to the forcing is expected to be dominated by waves at the forcing frequency. The "low,narrow" topography has $R_{L}>1$ for all but the lowest forcing velocity, while the "tall,narrow" topography has $R_{L}>1$ for the three largest values of forcing. We therefore expect to see higher harmonics generated for these two narrow topographies at the higher values of forcing.

The Froude number is always less than unity for the tall topographies, so that the topography presents a barrier to the flow, while for the low topographies $F r$ is greater than unity for the two largest values of forcing, when the flow can easily move over the topographic barrier. Note that the "tall,narrow" topography is the only one where the combination $F r<1, R_{L}>1$ is possible, at the highest forcing velocities. This particular region of parameter space might be expected to have a special character, since a parcel of water would only move up and over the entire topographic obstruction in a tidal cycle if $R_{L}>1$ and hence experience the full extent of the topographic barrier implied by $\mathrm{Fr}<1$. We expect the flow to be highly nonlinear in this regime.

To summarize the nondimensional parameters, "low,wide" always has $\gamma, R_{L}<1$, with $\operatorname{Fr}>1$ for large $U_{0}$; "low,narrow" has $\gamma>1$ and $R_{L}, F r>1$ for large $U_{0}$; "tall,wide" has $\gamma>1$ and always has $F r, R_{L}<1$; and finally "tall,narrow" has $\gamma>1, F r<1$ and for large $U_{0}, R_{L}>1$. 
For the four different topographies, we are forced to use different combinations of vertical and horizontal resolution. For the two "low" topographies, resolution is much finer near the bottom, so as to resolve the small bumps. We also concentrate horizontal resolution around the topography, but ensure that the coarsest horizontal resolution, near the boundaries, is still sufficient to resolve the lengthscales of the propagating waves. Since the "low narrow" topography generates waves on much smaller horizontal scales, the domain size is forced to be much smaller. The "tall, narrow" topography has larger topographic lengthscales than the "low narrow", so the minimum $\Delta x$ is somewhat larger, but since narrow beams are also generated, the maximum $\Delta x$ is again limited. The two wide topographies have larger domain sizes, allowing the wave propagation to be observed more fully. Horizontal and vertical resolutions are shown for all 4 topographies in figure 2 .

\section{Results}

\subsection{Qualitative features}

A series of figures $(3,4,5,6)$ show snapshots of the baroclinic velocity field for low and high forcing $\left(U_{0}=2 \mathrm{~cm} / \mathrm{s}\right.$ and $\left.U_{0}=24 \mathrm{~cm} / \mathrm{s}\right)$, for the 4 different topographies, at the end of the calculation (11.2 tidal periods after the onset of forcing). To obtain the baroclinic velocity we have subtracted the barotropic velocity, obtained through a companion barotropic calculation, from the full velocity field. The full domain is shown in each case. For the "low, wide" topography (figure 3) a mode 1 internal wave is seen propagating in both directions away from the topographic feature. Increasing the amplitude of the forcing leads to no qualitative changes in the response. The "low, narrow" topography has a response dominated by the principal frequency at $U_{0}=2 \mathrm{~cm} / \mathrm{s}$, in the form of a narrow beam (figure 4a). As the amplitude of the forcing increases, responses at higher harmonics appear: the beam at a steeper angle for $U_{0}=8 \mathrm{~cm} / \mathrm{s}$ (figure $4 \mathrm{~b}$ ) corresponds to the $2 \omega_{0}$ internal tide. Unlike Lamb (2004) we do not see evidence for generation of harmonics by nonlinear wavewave interactionsat the locations where beams intersect: all our beams at higher frequencies can be traced back to the topography itself. For the tall topographies, upward and downward propagating beams are seen for $U_{0}=2 \mathrm{~cm} / \mathrm{s}$ (figures $5 \mathrm{a}, 6 \mathrm{a}$ ), while at $U_{0}=24 \mathrm{~cm} / \mathrm{s}$ the velocity field near the topography becomes more disorganized, particularly for the "tall, narrow" topography. 
Closeups of the temperature field at $U_{0}=24 \mathrm{~cm} / \mathrm{s}$ are shown for all four topographies in figure 7 at a time of maximum flow to the right. At low amplitudes of forcing (not shown) little deformation of isopycnals is visible. While for the "low, wide" topography only a small deflection is produced (figure 7a), for the "low, narrow" topography (figure 7b) the downward plunge of the isopycnals downstream of the topography is much more marked. Note that for oscillating flows a water parcel experiences a greater vertical deflection during the tidal period for narrower topography than for a wider topography of the same height. For the tall topographies, a downward plunge over the ridge is followed by a rebound and some overturning (figure 7c,d), with density inversions especially visible for the "tall, narrow" topography. These snapshots are suggestive of transient hydraulic behavior, although more rigorous analysis would be necessary to determine whether there is truly a transition from subcritical to supercritical flow.

\section{$3.2 \quad$ Frequency Spectra}

The vertical velocity frequency spectra for all 20 simulations are shown in figures 8 and 9, scaled by $U_{0}^{2}$. Both the spectra diagnosed from the numerical simulations, and the spectra predicted from Khatiwala (2003) are shown. Note that the power is shown on a logarithmic scale so that both large and small order of magnitude features are visible, while frequency is shown on a linear scale, since we are interested in highlighting the harmonics, which cover less than one order of magnitude in frequency. The diagnosed spectra confirm the observation that the response to the "low, wide" topography over the range of velocities studied is approximately linear - the peak at the forcing frequency is much larger than that at the higher harmonics, even for the maximum value of forcing. The predicted spectra are very close to the simulated spectra. For the "low narrow" topography, at $U_{0}=2 \mathrm{~cm} / \mathrm{s}$ the largest peak is at the forcing frequency with successively smaller subsidiary peaks at $2 \omega_{0}$, and $3 \omega_{0}$. For stronger forcing the harmonics become more important. Interestingly all higher harmonics have the same order of magnitude as the forcing frequency by $U_{0}=8 \mathrm{~cm} / \mathrm{s}$, and not just the first harmonic: again this agrees with the theoretical prediction. Our time sampling is only sufficient to resolve up to a frequency of $4 \omega_{0}$, although even higher frequency responses may be generated (note that $N / \omega_{0}=5.6$, so that propagating waves of frequency up to $5 \omega_{0}$ are possible), leading to aliasing which produces the energy seen at zero frequency.

The "tall wide" topography spectrum again has a dominant peak at the forcing frequency for 
$U_{0}=2 \mathrm{~cm} / \mathrm{s}$, and although this frequency continues to dominate, energy is seen at other frequencies for higher $U_{0}$. A curious result seen at $U_{0}=8 \mathrm{~cm} / \mathrm{s}$ is the peak at $4 \omega_{0}$, which disappears at higher forcing - the cause of this is unknown. This peak does not appear in the theoretical result, which predicts only small harmonic responses for the highest forcing. Additionally, the simulated spectra have much more power at intermediate frequencies, between the harmonic peaks, than the theoretical predictions.

The "tall, narrow" topography again shows a single dominant frequency response at $U_{0}=$ $2 \mathrm{~cm} / \mathrm{s}$ and multiple peaks at the harmonic frequencies at higher forcing, as predicted from the Bell (1975); Khatiwala (2003) theory. However, the harmonics gain equivalent magnitude to the forcing frequency peak at somewhat weaker forcing in the simulations compared to the predictions - this might be attributed to the enhancement of the barotropic flow above the topography due to the finite $\delta$. Note that in the spectra diagnosed from the "tall narrow" and to some extent the "tall, wide" simulations, the peaks are not discrete; significant energy is found at frequencies between the $n \omega_{0}$ harmonics. This contrasts with the spectra from the low topography simulations or the predicted spectra. The broadened peaks could result from Doppler shifting of the internal waves by the mean flow - however similar Doppler shifting would be expected for the low topographies, since the velocities in the deep water where these spectra are obtained are similar, but is absent. Hence the broadened peaks might indicate the development of a continuum such as in a breakdown to turbulence in the tall topography simulations.

To summarize these qualitative observations: the "low, wide" topography produces a linear response (a mode 1 internal wave at the forcing frequency) for all forcing examined here. The "low, narrow" topography produces beam-like internal waves at progressively more harmonic frequencies as the forcing is increased. The "tall narrow" forcing produces a double beam at the forcing frequency for weak forcing, becoming more disorganized and turbulent, although still with energetic peaks at the harmonics of the forcing frequency for stronger forcing. The predictions of the frequency of the response made by Bell (1975) and Khatiwala (2003) compare well with the numerical simulations, except for the broadening of the peaks in the tall topography simulations, which we attribute to nonlinear processes not accounted for by the theory. In the theoretical predictions the frequency of the wave response is determined by the generation process rather than any subsequent nonlinear wave interactions - the agreement between the simulations and the theory in this respect 
indicates similar processes are responsible for the appearance of the harmonics. Energy transfer between harmonics does not appear to play an important role except in the transfer of energy to frequencies which are not tidal harmonics in the tall topography simulations.

\subsection{Energy conversion and dissipation}

Many recent studies have made predictions for the rate at which energy is converted from the barotropic to the baroclinic field, and in particular the changes in this conversion rate introduced by topography of finite steepness $\gamma$ and finite height relative to the total depth $\delta$ (Balmforth et al., 2002; Llewellyn Smith and Young, 2002; Khatiwala, 2003; Llewellyn Smith and Young, 2003).

We can compare our numerical results with the theoretical predictions, and also examine the effect of increasing the amplitude of the forcing. We diagnose the conversion rate in the numerical simulations as done by Khatiwala (2003):

$$
P=\int_{x=0}^{x=L x} p^{\prime}(x, z=h(x), t) U_{b t}(x, t) \frac{d h}{d x} d x
$$

where $p^{\prime}(x, z=h(x), t)$ is the perturbation pressure at the height of the topography, given by

$$
p^{\prime}(x, z, t)=\rho_{0} g\left(\eta-\eta_{b t}\right)+p(x, z, t)-P_{0}(z)
$$

where $\eta$ is the free-surface elevation, $\eta_{b t}$ is the free-surface elevation in a companion barotropic calculation, $p(x, z, t)$ is the full pressure (including nonhydrostatic components) not including the free-surface contribution, and $P_{0}(z)$ is the hydrostatic reference pressure when the fluid is at rest. $U_{b t}$ is the velocity field from the companion barotropic calculation, and is not necessarily equal to the forcing velocity when there is large amplitude topography. The time-averaged conversion rate is shown in figure 10a for all 4 topographies, along with error bars indicating the size of the temporal fluctuations. (The error bars are not meant to indicate systematic numerical errors, which may be unaccounted for.) All values are shown scaled by $U_{0}^{2} h_{0}^{2}$.

For an infinitely deep ocean and shallow topography, Bell (1975) predicts a conversion rate of

$$
P_{\infty}=\frac{\pi}{8} \rho_{0} \frac{\left[\left(N^{2}-\omega_{0}^{2}\right)\left(\omega_{0}^{2}-f^{2}\right)\right]^{1 / 2}}{\omega_{0}} U_{0}^{2} h_{0}^{2}
$$

which is marked on the plot by a solid line. This prediction agrees well with the conversion rate diagnosed for the "low wide" topography. 
For steep topography, both St Laurent et al. (2003) and Llewellyn Smith and Young (2003) predict that for $\delta<<1$, knife-edge topography will have a conversion rate twice that of the subcritical topography. We do find that the "low narrow" topography has a conversion rate nearly twice that of the "low wide" topography (although of course $\gamma=2.0$ is far from an infinite slope). For $\delta=0.5$, St Laurent et al. (2003) predict a conversion rate of about $P_{\text {knife }}=3.2 P_{\text {witch, }, \gamma=1}=$ $0.58 \mathrm{kgm}^{-3} \mathrm{~s}^{-1} U_{0}^{2} h_{0}^{2}$. This is similar to that diagnosed for the "tall wide" topography (which it should be noted has $\gamma=2$, quite far from the $\gamma=\infty$ limit). Curiously the "tall narrow" topography has smaller conversion rate than the "tall wide" topography. In contrast, Khatiwala (2003) found numerically that the conversion rate increased monotonically with $\gamma$ for Gaussian topography; however he only considered $\gamma$ as high as 1.6, whereas our tall topographies have $\gamma=2$ and 8 respectively.

The two "wide" topographies have conversion rates which do not change significantly (after scaling by $U_{0}^{2}$ ) as the forcing amplitude increases. However, the "low narrow" topography has a scaled conversion rate which decreases as the forcing increases, so that for the highest amplitude forcing the conversion rate is nearly the same as for the "low wide" topography. By contrast, the "tall narrow" topography has a scaled conversion rate which is less than that of the "tall wide" topography at small amplitude forcing, and increases as the forcing amplitude increases. At the moment we do not have an explanation for these two differing trends with forcing amplitude.

Figure 10b shows the dissipation $\operatorname{rate}^{1} \epsilon=\nu_{i}\left(\partial u_{j} / \partial x_{i}\right)^{2}$, integrated over the volume, and scaled by $U_{0}^{2} h_{0}^{2}$. Note that different sized domains are used for the different calculations; however, most of the dissipation occurs close to the topography, so that the total dissipation is relatively insensitive to the domain size. The dissipation for the "low narrow" topography greatly exceeds that for the other topographies. The scaled dissipation appears to vary little as forcing amplitude is increased, except that the dissipation in the "tall narrow" topography increases at large $U_{0}$.

The ratio between the conversion rate and the dissipation rate is of especial interest, since parameterizations of tidal mixing need to know how much of the energy extracted from the barotropic tide is radiated away as waves and how much is dissipated locally. In the parameterization imple-

\footnotetext{
${ }^{1}$ Note that this form of the dissipation is equivalent to that in H.Tennekes and Lumley (1972) when non-divergence is assumed, as it is in these simulations, and only those components which cannot be written as the divergence of a flux are included
} 
mented in Simmons et al. (2003), it is assumed that $1 / 3$ of the energy is dissipated locally. From figure $10 \mathrm{c}$ we see that this is an overestimate for most of the topographies studied; $10 \%$ is more typical. However, for the "low narrow" topography, a large proportion of the energy is dissipated, increasing to nearly $90 \%$ at the highest amplitude forcing. The proportion of energy dissipated also increases with forcing amplitude for the "tall narrow" topography.

It is likely that the enhanced dissipation in the "low narrow" topography results from the smaller lengthscales of the waves generated in this case, as indicated in figure 11, showing the vertical velocity vertical wavenumber spectra as a function of vertical mode number. The "low narrow" topography is the only case for which the spectral peak is at higher mode numbers; the other three topographies show most energy at mode numbers 1 and 2. (Note that the spectra are only shown for the representative forcing velocity of $U_{0}=8 \mathrm{~cm} / \mathrm{s}$, spectra at other forcing velocities showed similar qualitative behavior). These spectra, diagnosed at several locations relative to the topography, also show that few changes in the spectra occur away from the topography, so that most of the dissipation therefore takes place near to the topography.

\subsection{Mixing}

We would like to examine how much diapycnal mixing is associated with the dissipation of energy after the conversion from barotropic to baroclinic flow. Most models of diapycnal mixing (e.g. Osborn (1980)) assume that a constant fraction of the energy dissipated is converted into potential energy through mixing. The most direct way to examine the total mixing therefore is through a time-series of total change in potential energy. We expect the potential energy to increase in response to diabatic mixing; however potential energy also changes adiabatically, as dense fluid is pushed up over topography by the flow, or due to passing internal waves, and hence a time-average over several cycles is necessary to obtain an accurate evaluation of the diabatic changes in potential energy. Unfortunately the open boundaries introduce a further complication - the total heat content is not preserved, due to density changes introduced at the boundaries. These spurious changes in heat content mask the potential energy changes generated by any mixing after a few tidal cycles. Attempts to measure the change in potential energy in the early part of the calculation (before the heat content changes are significant) were not conclusive due to the large noise associated with the tidal cycle. For these reasons diagnosing potential energy changes does not allow us to reach any 
conclusions regarding the diapycnal mixing.

The issue of the heat content changes introduced by the open boundaries makes it difficult to use other diagnostics, e.g. probability density functions of density or net changes in stratification, to draw conclusions about the diapycnal mixing. Furthermore, the 2-dimensional nature of the simulations and the low Reynolds numbers make it likely that mixing is underestimated in the simulations: for example we do not see any evidence for shear instability in the narrow beams which we might expect at higher Reynolds numbers. Additionally, at this resolution the implicit numerical diffusion associated with the advection scheme is large. For this reason, we emphasize that these are preliminary calculations, and further investigation of the mixing, involving higher resolution 3-D calculations will be necessary. These simulations should be at a resolution such that numerical diffusion is minimized compared to explicit diffusion. Improvements to the radiative boundary conditions may also be needed, since these currently appear to perform well in terms of allowing velocity signals out of the domain, but less well with the density signals, introducing spurious changes in total heat content.

\section{Discussion and conclusions}

In this survey of parameter space we have examined the generation of internal waves and dissipation produced by tidal flow over 4 different topographies. The "low wide" topography, with subcritical slope, small tidal excursion parameter and large $F r$ is similar to the overall Mid-Atlantic Ridge structure, and leads to the generation of linear internal waves dominated by the forcing frequency and the gravest vertical mode, with a rate of power conversion given by Bell (1975) and small dissipation. The "low, narrow" topography is similar in structure to the small-scale roughness elements of the Mid-Atlantic Ridge, and leads to internal waves with smaller vertical wavelengths and a higher rate of dissipation. The presence of higher modes has indeed been noted in the observations from the MAR region. St Laurent and Nash (2004) have proposed that the dissipation rates in the MAR and Hawaiian Ridge data can be reconciled by assuming that the dissipation rate depends on the energy content of the higher vertical modes, in agreement with our simulations. An additional feature of the response of the flow to very narrow topography (i.e. high $R_{L}$ ) in the

simulations is the appearance of higher harmonic frequencies, as predicted by Bell (1975), but not 
yet resolved in the observational record. The "tall wide" topography shows a response dominated by the gravest vertical mode and the forcing frequency: similar behavior is seen observations from the Hawaiian Ridge (Klymak et al., 2005), which has a similar topographic structure. At the Hawaiian Ridge about $10 \%$ of the energy converted from the barotropic tide is found to be dissipated locally - this again agrees with our simulations for the "tall wide" topography. Finally, the "tall narrow" topography, which has large $R_{L}$ at the largest magnitude forcing, develops responses at the higher harmonic frequencies, with much broader peaks than for the "low narrow" topography. Along with observations of overturning features possibly associated with hydraulic jumps, these lead us to believe the "tall narrow" scenario is the most conducive to mixing. Similar hydraulic features have been seen in observations in Knight Inlet (Klymak and Gregg, 2004).

The simulations compare well with theoretical predictions in many respects: For the "low wide" topography the rate of energy conversion is well predicted by Bell (1975), which assumes subcritical slopes, as in this case. For the supercritical cases, the energy conversion is greater: for the "low narrow" case it approaches twice the subcritical value, as predicted by St Laurent et al. (2003) and Llewellyn Smith and Young (2003) for a knife-edge ridge. The energy conversion rate for the "tall wide" topography approaches that predicted for a knife-edge ridge of that height by St Laurent et al. (2003), while that for the "tall narrow" topography is smaller, but increases with forcing amplitude. The appearance of higher harmonics is in general well predicted by the Bell (1975) theory.

From these results we see that the net effect of tidal flow over topography depends not only on the height of the topography, but also on its width and its ability to block the flow, as measured by Fr. Narrower topography will lead to higher vertical modes and greater local dissipation, compared to wider topography of the same height or of the same steepness. Narrow topography which blocks the flow (high $R_{L}$ combined with low $F r$ ) can lead to hydraulic behavior conducive to overturning - although this scenario is probably found in coastal regions rather than the deep ocean.

These preliminary simulations provide motivation to examine mixing more closely, especially in the "tall narrow" and "low narrow" scenarios, through higher resolution 3-dimensional simulations. A more detailed study of the mixing must examine the dependence of mixing on the model's diffusive parameterization and advection scheme. Numerous other parameters, not varied in this study, such as stratification, tidal frequency (including forcing at multiple frequencies) and Coriolis parameter, 
may also modify the mixing.

\section{Acknowledgments}

KH was supported by a Summer Student Fellowship at Woods Hole Oceanographic Institution. SL was supported by Office of Naval Research grant N00014-03-1-0336. We would like to thank Samar Khatiwala for sharing his configuration of the MITgcm which served as the starting point for these simulations. We thank Jody Klymak, Brian Arbic and Robert Hallberg for their helpful comments and suggestions. Comments of three anonymous reviewers greatly helped to improve the manuscript.

\section{References}

Balmforth, N., Ierley, G., Young, W., 2002. Tidal conversion by subcritical topography. J. Phys. Oceanogr. 32, 2900-2914.

Bell, T., 1975. Topographically generated internal waves in the open ocean. J. Geophys. Res. 80, $320-327$.

Holloway, P., Merrifield, M., 1999. Internal tide generation by seamounts, ridges, and islands. J. Geophys. Res. 108, 25937-25951.

H.Tennekes, Lumley, 1972. A first course in turbulence.

Khatiwala, S., 2003. Generation of internal tides in an ocean of finite depth: analytical and numerical calculations. Deep Sea Res. 50, 3-21.

Klymak, J., Gregg, M., 2004. Tidally generated turbulence over the Knight Inlet Sill. J. Phys. Oceanogr. 34, 1135-1151.

Klymak, J., Moum, J., Nash, J. D., Kunze, E., Girton, J., Carter, G., Lee, C., Sanford, T., Gregg, M., 2005. An estimate of tidal energy lost to turbulence at the Hawaiian Ridge. J. Phys. Oceanogr. in press. 
Lamb, K., 2004. Nonlinear interaction among internal wave beams generated by tidal flow over supercritical topography. Geophys. Rev. Lett. 31, doi:10.1029/2003GL019393.

Legg, S., 2004. Internal tides generated on a corrugated continental slope. Part II: Along-slope barotropic forcing. J. Phys. Oceanogr. 34, 1824-1834.

Legg, S., Adcroft, A., 2003. Internal wave breaking at concave and convex continental slopes. J. Phys. Oceanogr. 33, 2224-2246.

Llewellyn Smith, S., Young, W., 2002. Conversion of the barotropic tide. J. Phys. Oceanogr. 32, $1554-1566$.

Llewellyn Smith, S., Young, W., 2003. Tidal conversion at a very steep ridge. J. Fluid. Mech. 495, $175-191$.

Marshall, J., Adcroft, A., Hill, C., Perelman, L., Heisey, C., 1997. A finite-volume, incompressible Navier-Stokes model for studies of the ocean on parallel computers. J. Geophys. Res. 102 (C3), $5753-5766$.

Munk, W., Wunsch, C., 1998. Abyssal recipes - II: energetics of tidal and wind mixing. Deep Sea Res. 45, 1977-2010.

Munroe, J. R., Lamb, K., 2005. Topographic amphitude dependence of internal wave generation by tidal forcing over idealized three-dimensional topography. J. Geophys. Res. 110, 10.1029/2004JC002537.

Nash, J., Kunze, E., Toole, J., Schmitt, R., 2004. Internal Tide Reflection and Turbulent Mixing on the Continental Slope. J. Phys. Oceanogr. 34, 1117-1134.

Nycander, J., 2005. Generation of internal waves in the deep ocean by tides. J. Geophys. Res. in press.

Osborn, T., 1980. Estimates of the local rate of vertical diffusion from dissipation measurements. J. Phys. Oceanogr. 10, 83-89.

Pietrzak, J., 1998. The use of TVD Limiters for Forward-in-Time Upstream-Biased Advection Schemes in Ocean Modeling. Mon. Wea. Rev. 126, 812-830. 
Polzin, K., 2004. Idealized solutions for the energy balance of the finescale internal wave field. J. Phys. Oceanogr. 34, 231-246.

Simmons, H., Jayne, S., St Laurent, L., Weaver, A., 2003. Tidally driven mixing in a numerical model of the ocean general circulation. Ocean Modelling 6, 245-263.

St Laurent, L., Nash, J., 2004. An examination of the radiative and dissipative properties of deep ocean internal tides. Deep Sea Res. 51, 3029-3042.

St Laurent, L., Stringer, S., Garrett, C., Perrault-Joncas, D., 2003. The generation of internal tides at abrupt topography. Deep Sea Res. 50, 987-1003.

Y.V. Lvov, K.L. Polzin, E. T., 2004. Energy spectra of the ocean's internal wave field: theory and observations. Physics Rev. Lett. 92, Art. No. 128501.

Zikanov, O., Slinn, D., 2001. Along-slope current generation by obliquely incident internal waves. J. Fluid. Mech. 445, 235-261. 


\section{List of Figures}

1 (a) The Froude number $F r=U_{0} /\left(N h_{0}\right)$ and (b) the tidal excursion parameter $R_{l}=$ $U_{0} /\left(\omega_{0} L\right)$ as a function of $U_{0}$ for the 4 different topographies. . . . . . . . . . 21

2 (a) The horizontal resolution $\Delta x$ as a function of $x$ (distance from the topographic peak) and (b) the vertical resolution $\Delta z$ as a function of $z$ for the four different topographic scenarios. . . . . . . . . . . . . . . . . . . . . . . . . . . . 22

3 Snapshots of the baroclinic velocity field $(\mathrm{m} / \mathrm{s})$ for the "low wide" topography, shown for (a) $\mathrm{U} 0=2 \mathrm{~cm} / \mathrm{s}$ and $(\mathrm{b}) \mathrm{U} 0=24 \mathrm{~cm} / \mathrm{s} \ldots \ldots . \ldots 22$

4 Snapshots of the baroclinic velocity field $(\mathrm{m} / \mathrm{s})$ for the "low narrow" topography, shown for (a) $U 0=2 \mathrm{~cm} / \mathrm{s}$, (b) $\mathrm{U} 0=8 \mathrm{~cm} / \mathrm{s}$, (c) $\mathrm{U} 0=16 \mathrm{~cm} / \mathrm{s}$ and (d) $\mathrm{U} 0=24 \mathrm{~cm} / \mathrm{s} \ldots 23$

5 Snapshots of the baroclinic velocity field $(\mathrm{m} / \mathrm{s})$ for the "tall wide" topography, shown for (a) $\mathrm{U} 0=2 \mathrm{~cm} / \mathrm{s}$ and $(\mathrm{b}) \mathrm{U} 0=24 \mathrm{~cm} / \mathrm{s} \ldots \ldots \ldots \ldots \ldots$

6 Snapshots of the baroclinic velocity field $(\mathrm{m} / \mathrm{s})$ for the "tall narrow" topography, shown for (a) $\mathrm{U} 0=2 \mathrm{~cm} / \mathrm{s}$ and (b) $\mathrm{U} 0=24 \mathrm{~cm} / \mathrm{s} \ldots \ldots \ldots \ldots 2$

7 Snapshots of the temperature field for the 4 different topographies, shown for U0 $=24 \mathrm{~cm} / \mathrm{s}$, at a time of maximum flow toward the right. Note that both the spatial scales and temperature scales are different in each image: in (a) and (b) we focus on the bottom $20 \%$ of the domain, since finite amplitude displacements are confined to that region because of the small height of the topography, while in (c) and (d) the full domain height is shown. The displacements in (a) and (b) are much smaller than in (c) and (d), and would not be visible if the same color scale as in (c) and (d) was used. . . .

8 The vertical velocity power spectrum, integrated over the whole depth, scaled by $U_{0}^{2}$, for (a) the "low wide" topography, $11 \mathrm{~km}$ away from the topographic peak, and (b) the "low narrow" topography, 3.6km away from the topographic peak. (i) The diagnosed spectrum from the numerical simulations; (ii) The predicted spectrum from Khatiwala's (2003) finite depth extension of Bell's (1975) theory. The predicted spectrum has been calculated by sampling the predicted vertical velocity field at the same frequency as the numerical simulations, and taking the fourier spectrum of that discretely sampled time-series. . . . . . . . . . . . . . . . . . . 26 
9 The vertical velocity power spectrum, as in figure 8, but for (a) the "tall wide" topography and (b) the "tall narrow" topography, both at a distance of $11 \mathrm{~km}$ from the topographic peak. . . . . . . . . . . . . . . . . . .

10 (a) The total power conversion from barotropic to baroclinic mode, scaled by $U_{0}^{2} h_{0}^{2}$, (b) the total dissipation within the integration volume scaled by $U_{0}^{2} h_{0}^{2}$, (c) the ratio of dissipation to conversion. Green = "low, wide", Red = "low, narrow", Blue = "tall, wide", Black = "tall, narrow". Also shown in (a) are Bell's (1975) prediction for the conversion rate for $200 \mathrm{~m}$ topography in infinitely deep fluid (solid), St Laurent et al's (2003) prediction for the conversion rate for a knife-edge ridge in infinitely deep fluid (dashed), and St Laurent et al's (2003) prediction for the conversion rate for a knife-edge ridge with $\delta=0.5$ (dotted) . . . . . . . . . . . . . . . .

11 The vertical velocity spectrum, normalized by the forcing velocity, shown as a function of vertical mode number, calculated at 3 different locations relative to the topographic peak, shown for the 4 different topographies: (a) "low wide", (b) "low narrow", (c) "tall wide", (d) "tall narrow". In all cases the forcing velocity is $U_{0}=8 \mathrm{~cm} / \mathrm{s}$ and the spectra are time-averaged. . . . . . . . . . . . . . . 


\begin{tabular}{|c|c|c|c|c|}
\hline Dimensional Parameter & "low, wide" & "low,narrow" & "tall,wide" & "tall,narrow" \\
\hline & & & & \\
$h_{0}$ & $200 \mathrm{~m}$ & $200 \mathrm{~m}$ & $2350 \mathrm{~m}$ & $2350 \mathrm{~m}$ \\
$L$ & $10000 \mathrm{~m}$ & $413 \mathrm{~m}$ & $4857 \mathrm{~m}$ & $1215 \mathrm{~m}$ \\
$\gamma=d h / d x_{\max } / s$ & 0.0827 & 2.00 & 2.00 & 8.00 \\
$h_{0} / H$ & 0.0426 & 0.0426 & 0.5 & 0.5 \\
\hline
\end{tabular}

Table 1: Values of topographic parameters

(a)

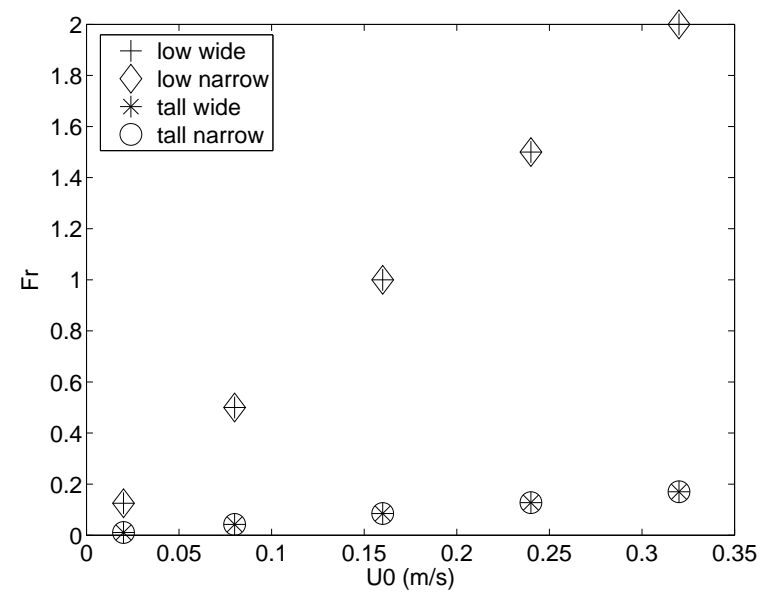

(b)

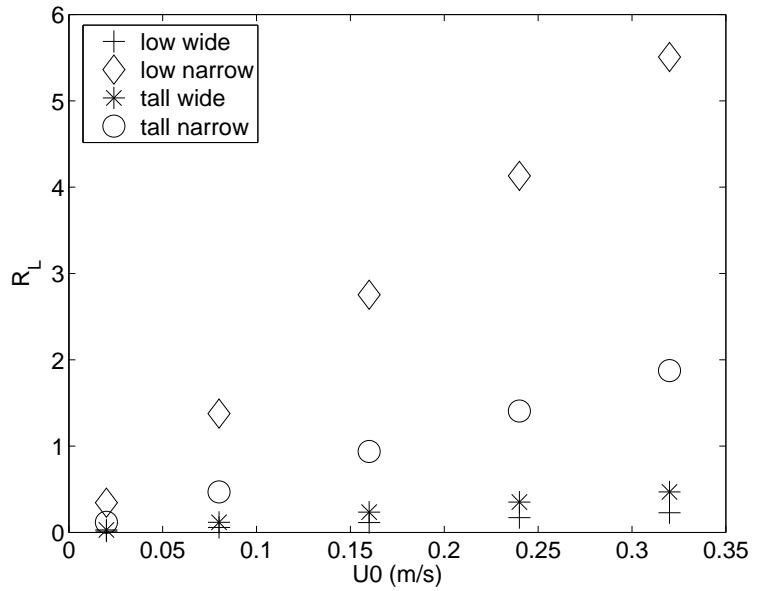

Figure 1: (a) The Froude number $F r=U_{0} /\left(N h_{0}\right)$ and (b) the tidal excursion parameter $R_{l}=$ $U_{0} /\left(\omega_{0} L\right)$ as a function of $U_{0}$ for the 4 different topographies. 
(a)

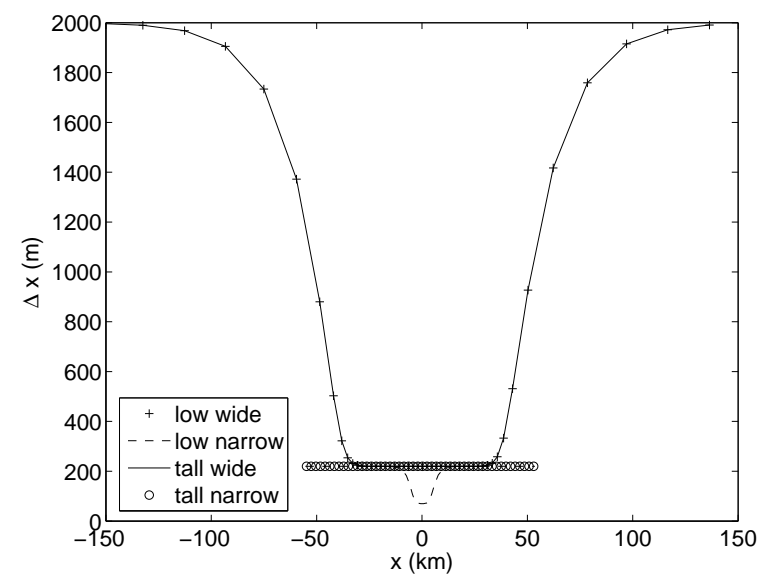

(b)

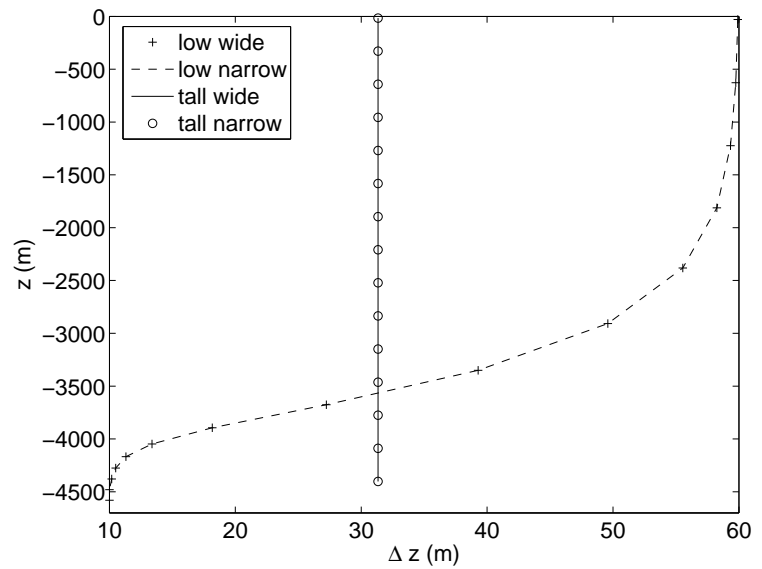

Figure 2: (a) The horizontal resolution $\Delta x$ as a function of $x$ (distance from the topographic peak) and (b) the vertical resolution $\Delta z$ as a function of $z$ for the four different topographic scenarios.

(a)

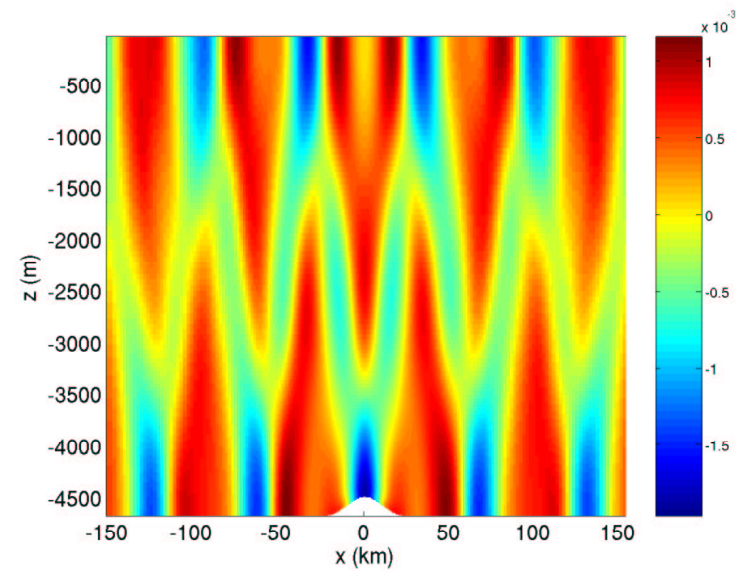

(b)

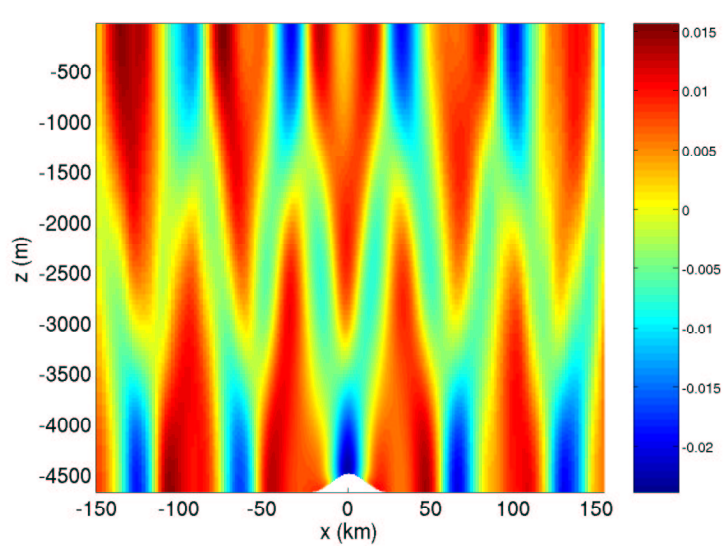

Figure 3: Snapshots of the baroclinic velocity field $(\mathrm{m} / \mathrm{s})$ for the "low wide" topography, shown for (a) $\mathrm{U} 0=2 \mathrm{~cm} / \mathrm{s}$ and (b) $\mathrm{U} 0=24 \mathrm{~cm} / \mathrm{s}$ 
(a)

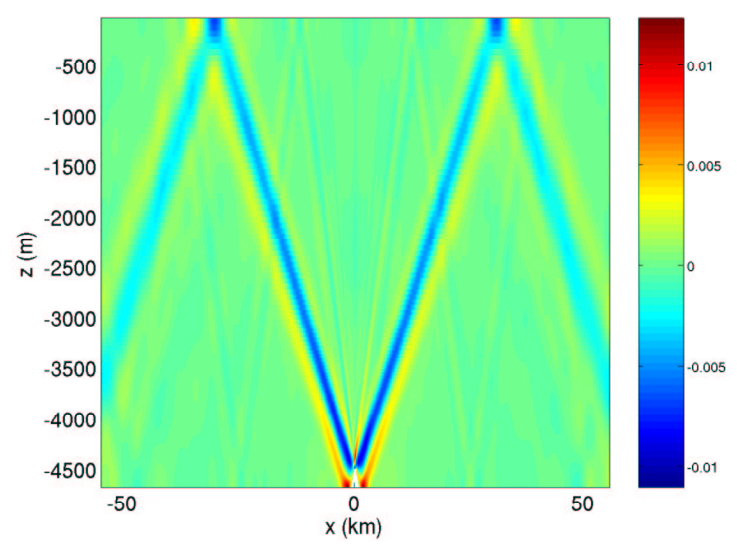

(c)

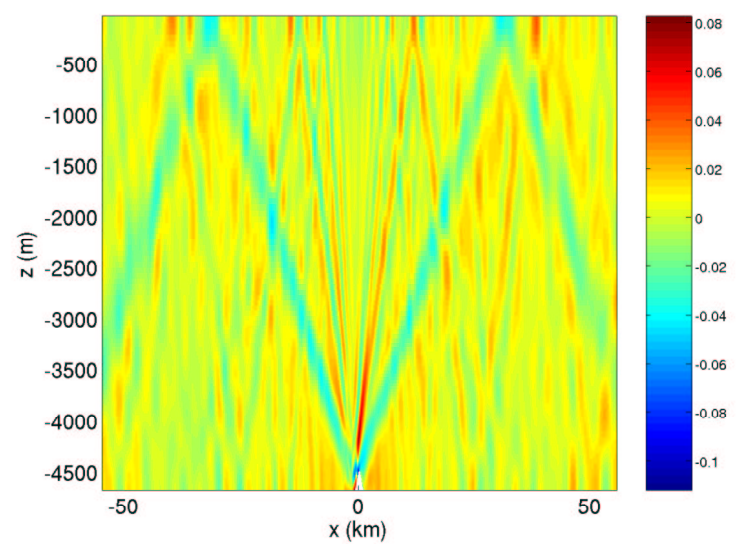

(b)

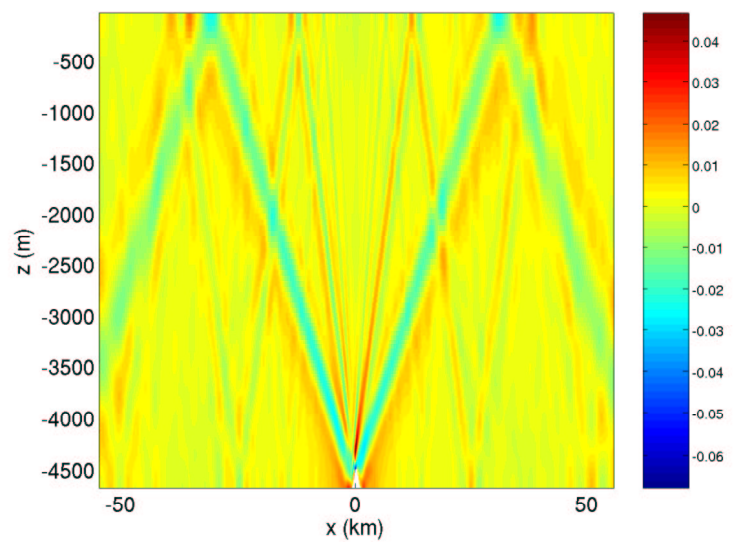

(d)

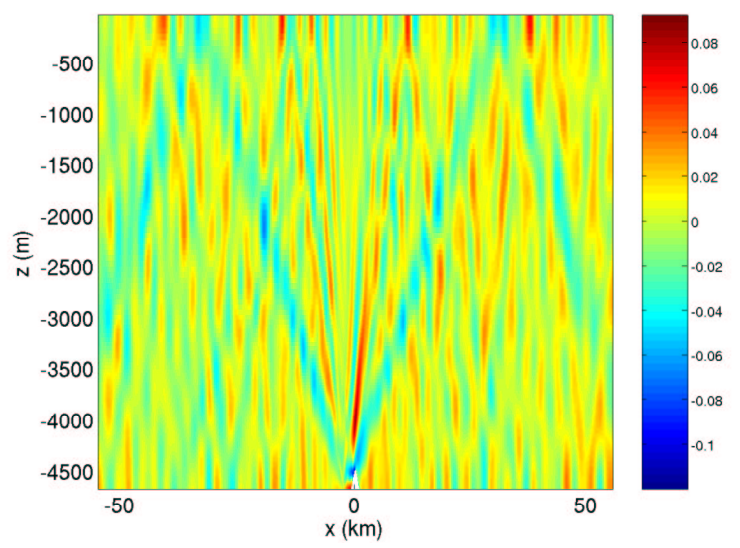

Figure 4: Snapshots of the baroclinic velocity field $(\mathrm{m} / \mathrm{s})$ for the "low narrow" topography, shown for (a) $\mathrm{U} 0=2 \mathrm{~cm} / \mathrm{s},(\mathrm{b}) \mathrm{U} 0=8 \mathrm{~cm} / \mathrm{s},(\mathrm{c}) \mathrm{U} 0=16 \mathrm{~cm} / \mathrm{s}$ and $(\mathrm{d}) \mathrm{U} 0=24 \mathrm{~cm} / \mathrm{s}$ 
(a)

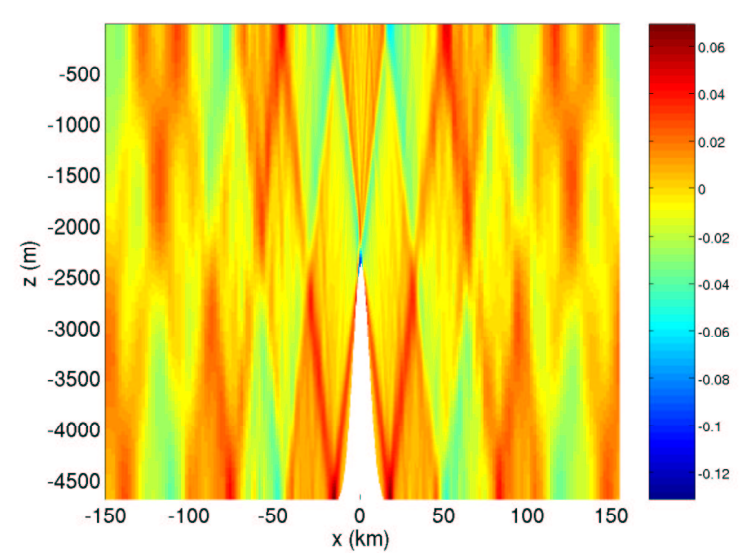

(b)

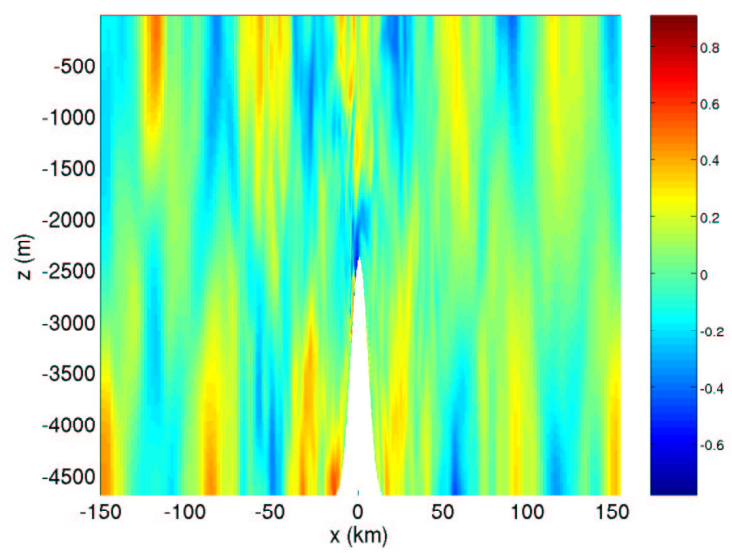

Figure 5: Snapshots of the baroclinic velocity field $(\mathrm{m} / \mathrm{s})$ for the "tall wide" topography, shown for (a) $\mathrm{U} 0=2 \mathrm{~cm} / \mathrm{s}$ and (b) $\mathrm{U} 0=24 \mathrm{~cm} / \mathrm{s}$

(a)

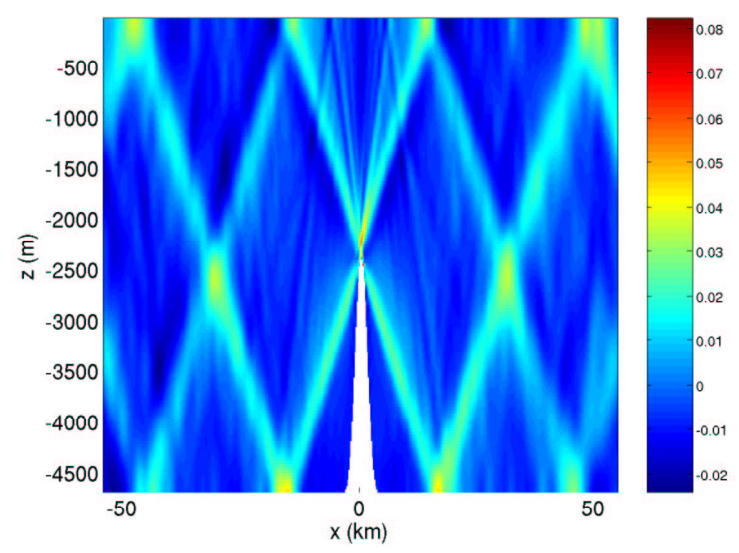

(b)

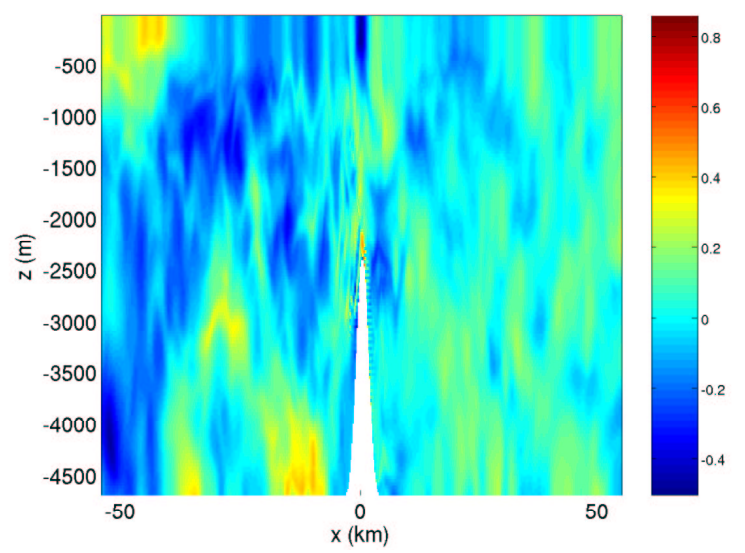

Figure 6: Snapshots of the baroclinic velocity field $(\mathrm{m} / \mathrm{s})$ for the "tall narrow" topography, shown for (a) $\mathrm{U} 0=2 \mathrm{~cm} / \mathrm{s}$ and (b) $\mathrm{U} 0=24 \mathrm{~cm} / \mathrm{s}$ 
(a)

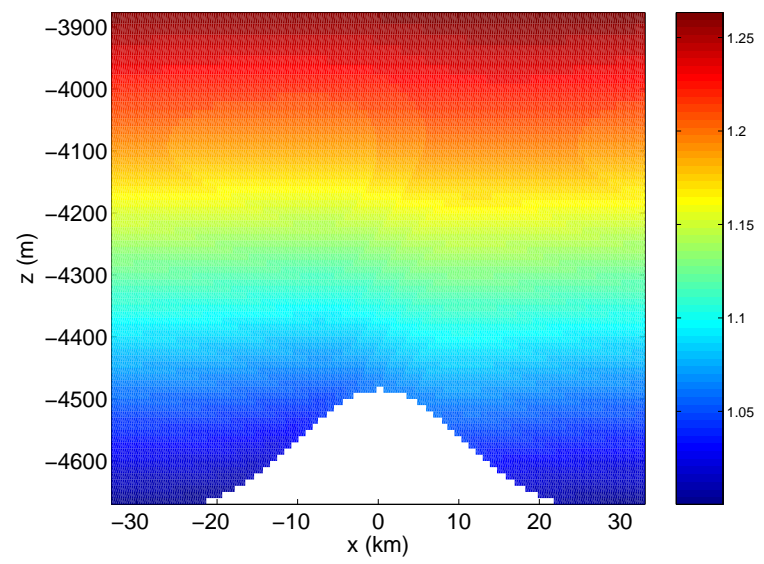

(c)

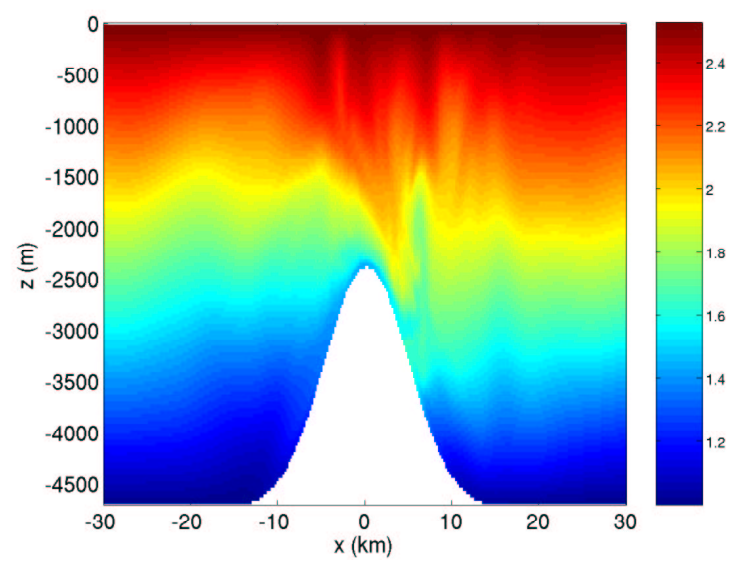

(b)

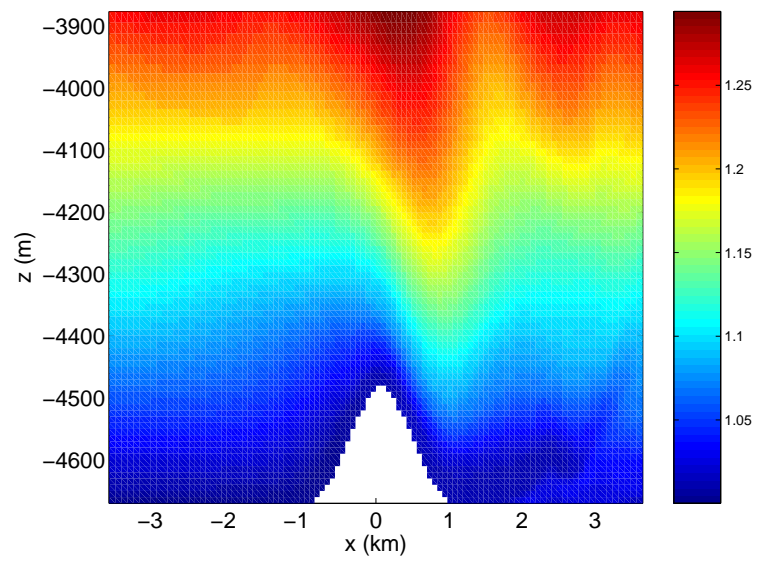

(d)

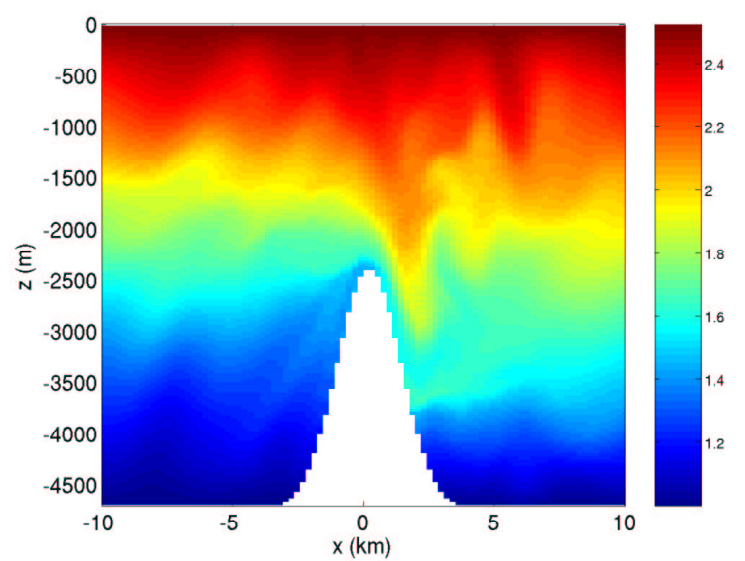

Figure 7: Snapshots of the temperature field for the 4 different topographies, shown for U0=24cm $/ \mathrm{s}$, at a time of maximum flow toward the right. Note that both the spatial scales and temperature scales are different in each image: in (a) and (b) we focus on the bottom $20 \%$ of the domain, since finite amplitude displacements are confined to that region because of the small height of the topography, while in (c) and (d) the full domain height is shown. The displacements in (a) and (b) are much smaller than in (c) and (d), and would not be visible if the same color scale as in (c) and (d) was used. 
(a) "low wide"

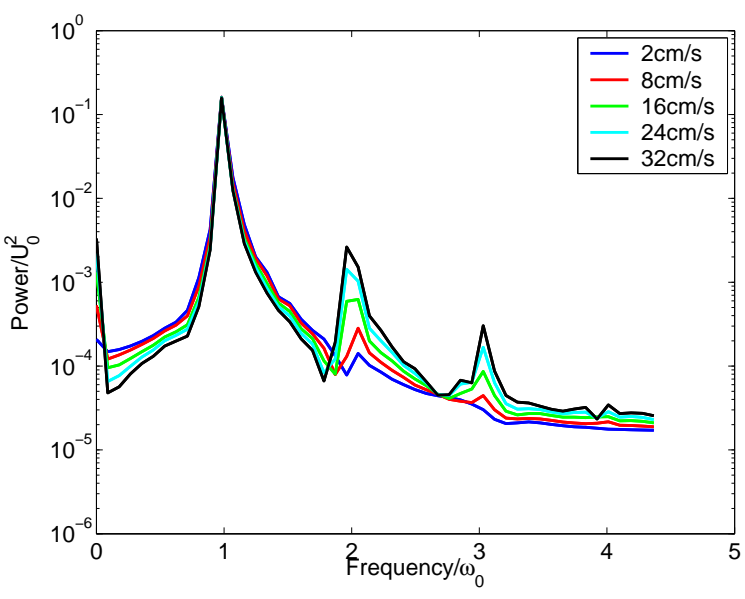

(i)

(ii)

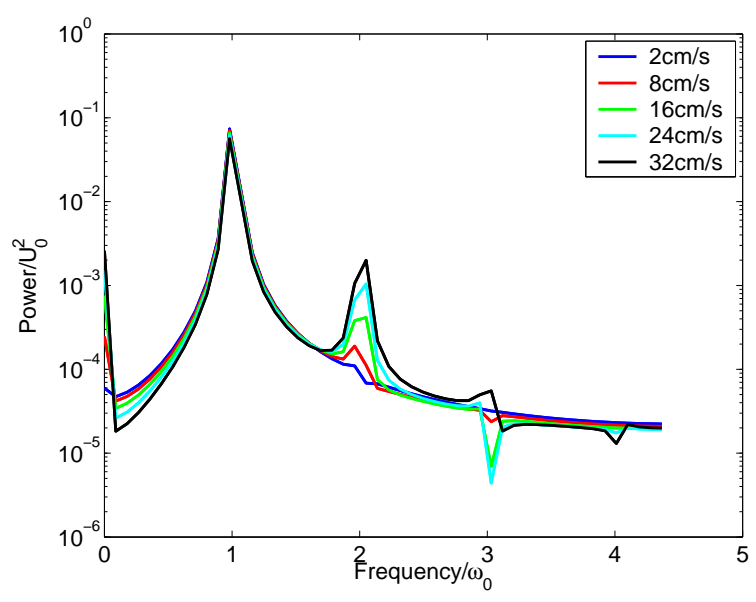

(b) "low narrow"
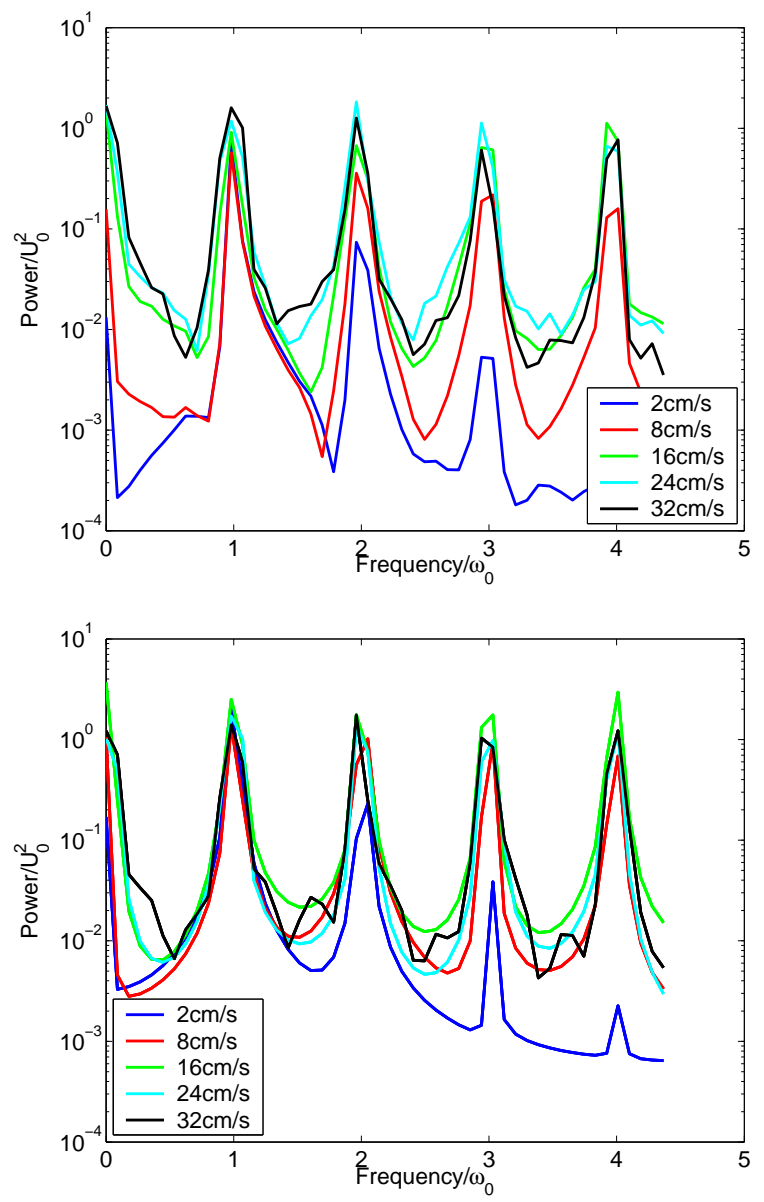

Figure 8: The vertical velocity power spectrum, integrated over the whole depth, scaled by $U_{0}^{2}$, for (a) the "low wide" topography, $11 \mathrm{~km}$ away from the topographic peak, and (b) the "low narrow" topography, 3.6km away from the topographic peak. (i) The diagnosed spectrum from the numerical simulations; (ii) The predicted spectrum from Khatiwala's (2003) finite depth extension of Bell's (1975) theory. The predicted spectrum has been calculated by sampling the predicted vertical velocity field at the same frequency as the numerical simulations, and taking the fourier spectrum of that discretely sampled time-series. 
(a) "tall wide"

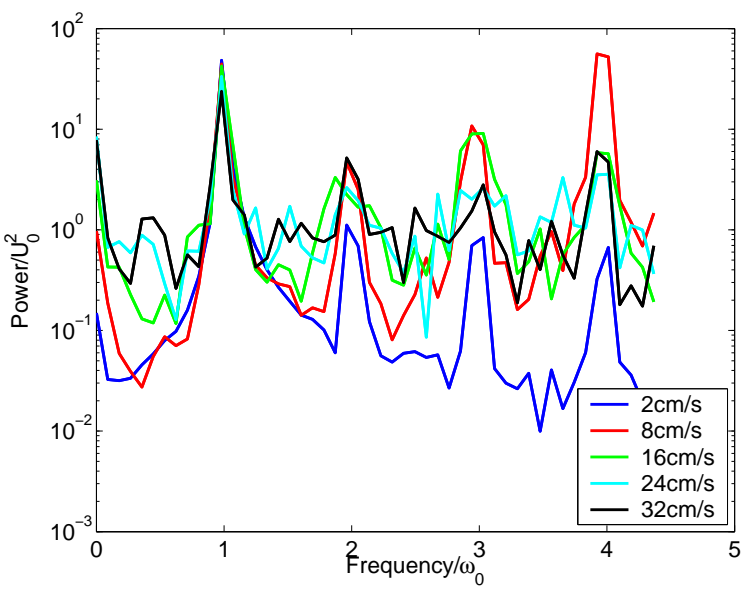

(i)

(ii)

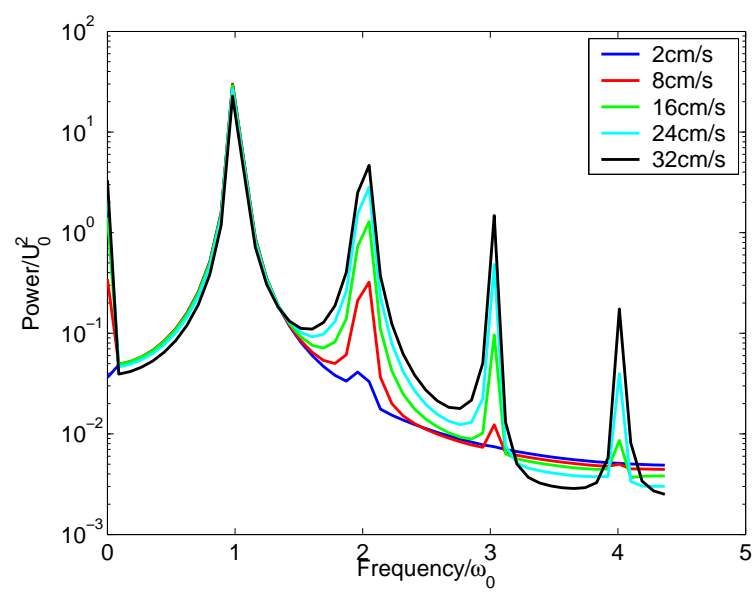

(b) "tall narrow"
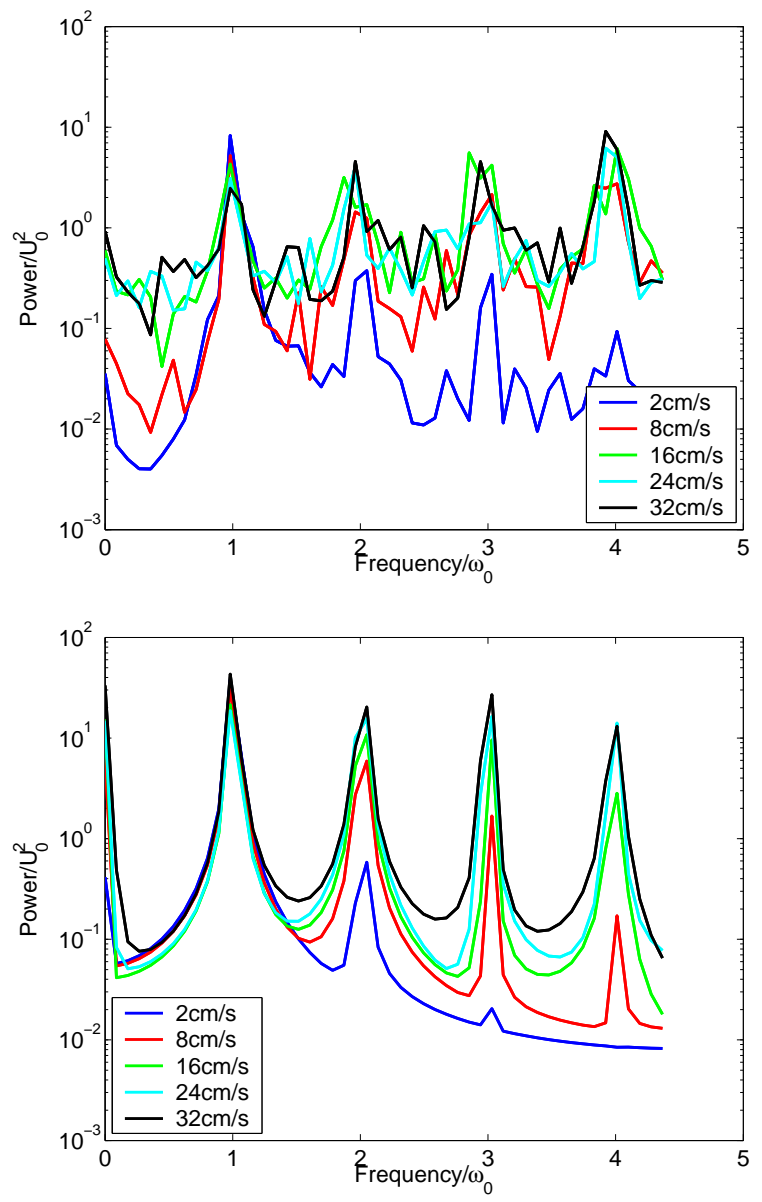

Figure 9: The vertical velocity power spectrum, as in figure 8, but for (a) the "tall wide" topography and (b) the "tall narrow" topography, both at a distance of $11 \mathrm{~km}$ from the topographic peak. 
(a) Conversion

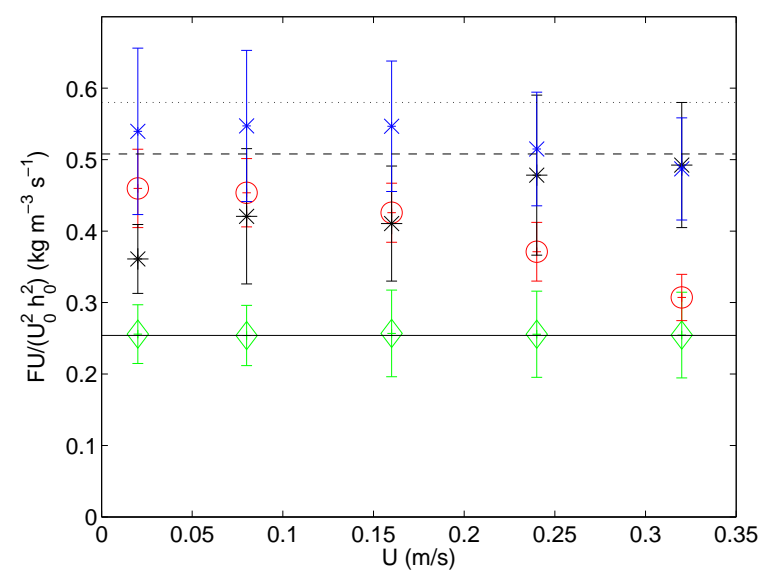

(b) Dissipation

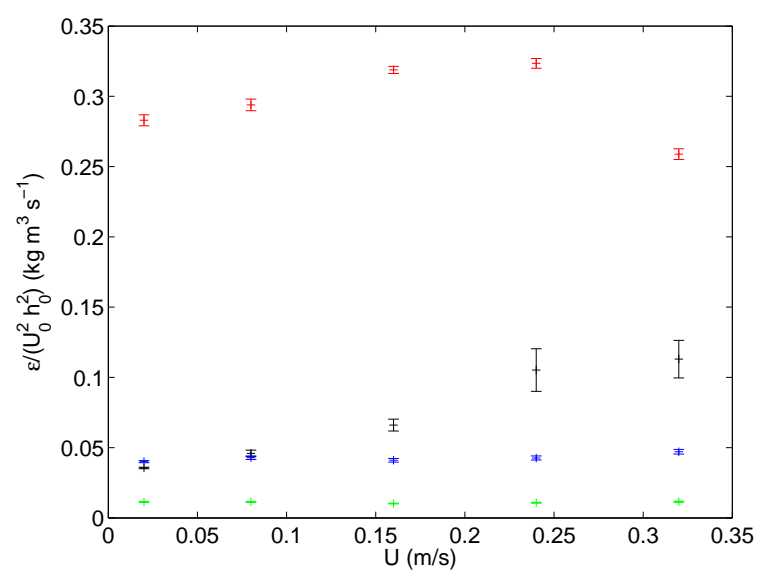

(c) Ratio

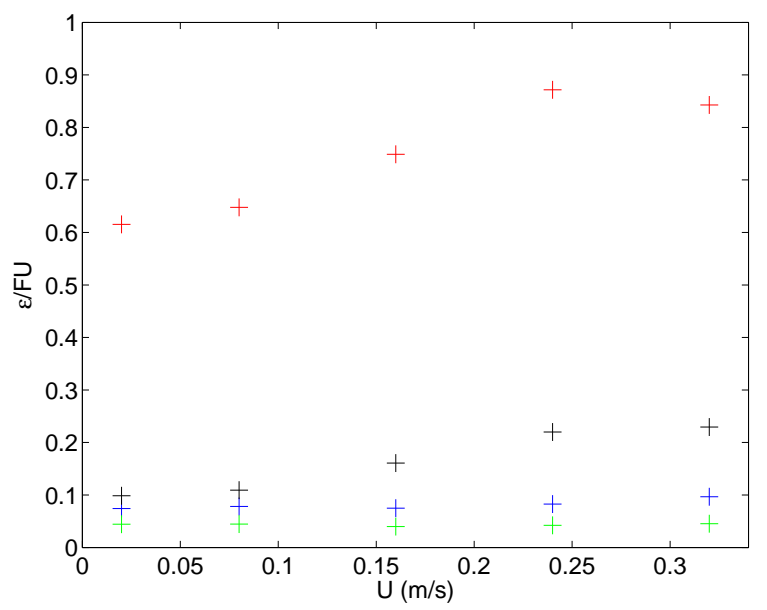

Figure 10: (a) The total power conversion from barotropic to baroclinic mode, scaled by $U_{0}^{2} h_{0}^{2}$, (b) the total dissipation within the integration volume scaled by $U_{0}^{2} h_{0}^{2}$, (c) the ratio of dissipation to conversion. Green = "low, wide", Red = "low, narrow", Blue = "tall, wide", Black = "tall, narrow". Also shown in (a) are Bell's (1975) prediction for the conversion rate for 200m topography in infinitely deep fluid (solid), St Laurent et al's (2003) prediction for the conversion rate for a knife-edge ridge in infinitely deep fluid (dashed), and St Laurent et al's (2003) prediction for the conversion rate for a knife-edge ridge with $\delta=0.5$ (dotted). 
(a)

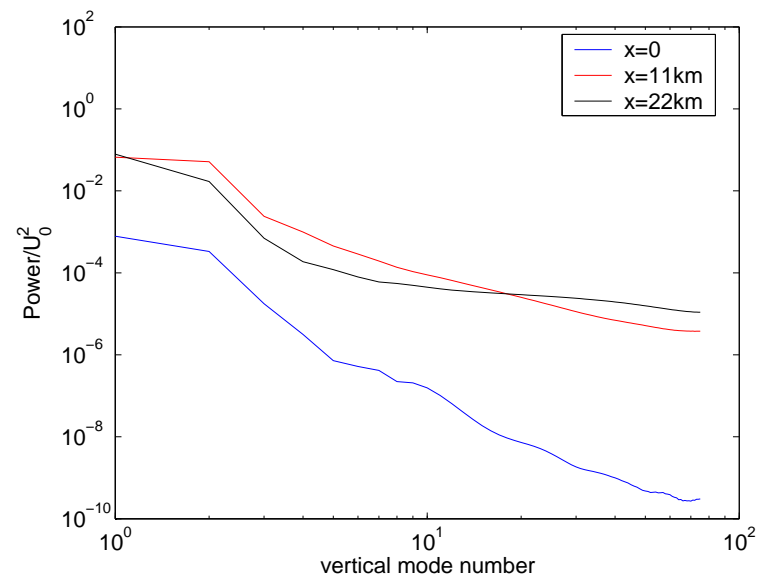

(c)

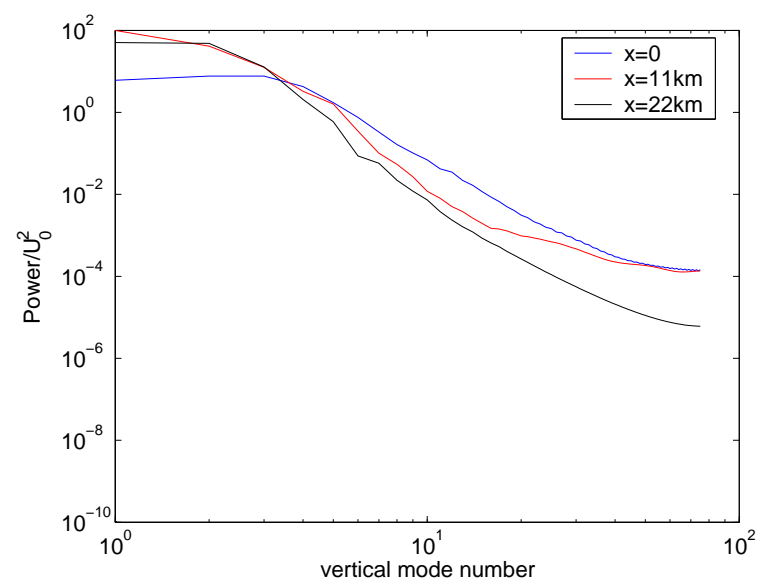

(b)

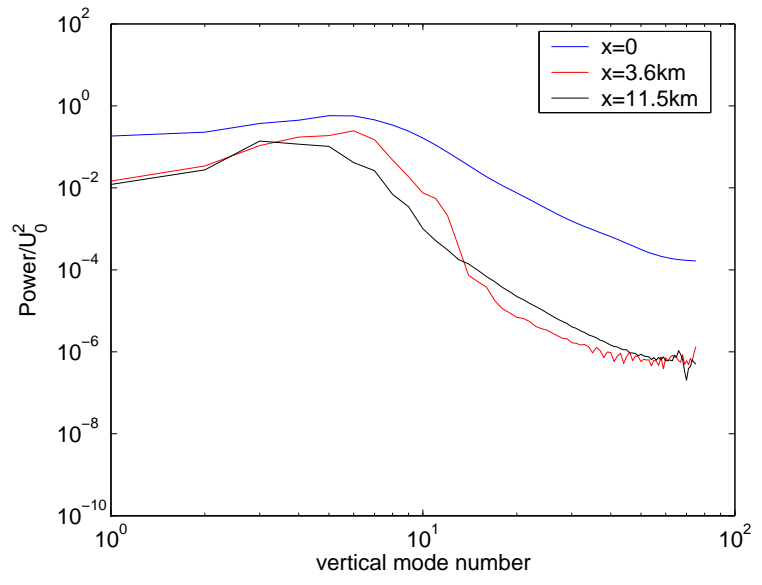

(d)

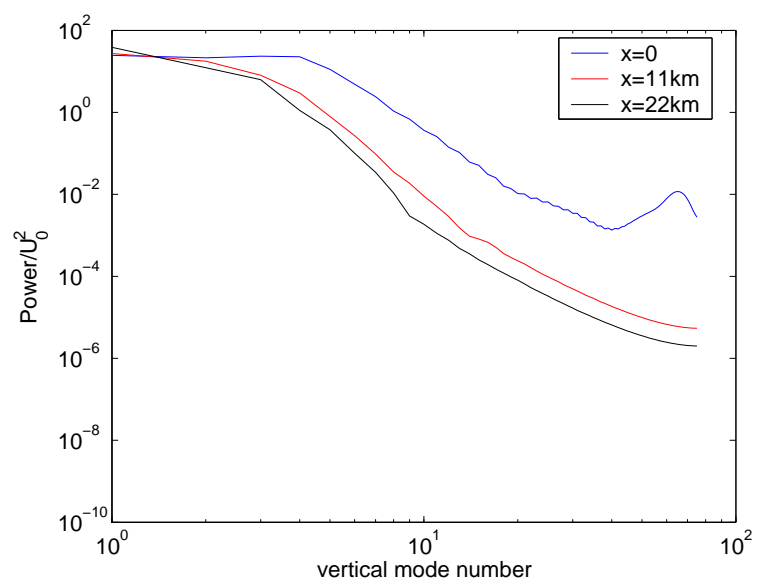

Figure 11: The vertical velocity spectrum, normalized by the forcing velocity, shown as a function of vertical mode number, calculated at 3 different locations relative to the topographic peak, shown for the 4 different topographies: (a) "low wide", (b) "low narrow", (c) "tall wide", (d) "tall narrow". In all cases the forcing velocity is $U_{0}=8 \mathrm{~cm} / \mathrm{s}$ and the spectra are time-averaged. 